\title{
How useful are clinical guidelines for the management of obesity in general practice?
}

\author{
Stewart Mercer
}

\begin{abstract}
Obesity is a major public health issue and numerous clinical guidelines have been published to support management. One of the most comprehensive guidelines on obesity was published by the National Institute for Health and Clinical Excellence (NICE) in 2006 (NICE guideline 43) which aims to offer practical recommendations based on the available evidence and has a strong focus on primary care both in terms of prevention and clinical care. The current article summarises these guidelines in relation to primary care, reports on new evidence and developments since they were published, and critically appraises the usefulness of guidelines for management of obesity.
\end{abstract}

$S$ Mercer, PhD, FRCGP, professor of primary care research general practice and primary care, Division of CommunityBased Sciences, University of Glasgow, Glasgow.

Address for correspondence

Stewart W Mercer, Division of Community-Based Sciences, University of Glasgow, 1 Horselethill Road, Glasgow G12 9LX. Email: s.mercer@clinmed.gla.ac.uk

Submitted: 20 April 2009; Editor's response: 11 June 2009; final acceptance: 6 October 2009.

OBritish Journal of General Practice 2009; 59: 863-868. DOI: 10.3399/bjgp09X472917

\section{BACKGROUND}

Obesity is a global public health issue, and the UK is no exception to this world epidemic, with major increases in obesity and overweight in both adults and children reported in recent decades. The National Institute for Health and Clinical Excellence (NICE) Clinical Guideline 43 on the prevention, identification, assessment, and management of overweight and obesity in adults and children, published in 2006, is a detailed, thorough, and ambitious clinical guideline on the topic. ${ }^{1}$ Chronic disease management in many countries, including the UK, is increasingly being delivered in general practice and primary care using protocols based on guidelines. ${ }^{2,3}$ Why not add obesity to the growing list of conditions now being treated by such an approach? General practice is, in principle, an obvious setting in countries with well-developed primary care systems such as in the UK, given the population coverage this provides. Virtually the whole population in the UK is registered with a practice and approximately three-quarters see their GP during a year, rising to $90 \%$ in 5 years. What then do NICE guidelines say about obesity management in primary care? What major new evidence and developments have taken place since they were published 3 years ago? And what questions remain unanswered about the management of obesity in general practice and primary care?

\section{NICE CLINICAL GUIDELINE 43}

Obesity: the prevention, identification, assessment, and management of overweight and obesity in adults and children

The guidelines are presented in a number of different documents; healthcare professionals can read the full guideline (divided into seven sections plus 16 appendices amounting to 1942 pages) or a summary version (80 pages including appendices) or two quick reference guides, one of which is intended for the NHS (27 pages). There are additional documents for patients, carers, and the public, as well as background and guidance on implementing the guidance. 


\section{How this fits in}

Obesity is a major public health concern internationally and in the UK. Clinical guidelines on obesity published by NICE in 2006 place a key focus on primary care. Although the NICE guidelines on obesity offer a comprehensive distillation of the international literature, there is a paucity of studies carried out in primary care or in the UK. In addition, the complex nature of obesity and the high prevalence of comorbidity mitigate against the simple application of guidelines in primary care.

The coverage, aims, and priorities of the guideline are shown in Box 1. NICE guidelines contain integrated public health and clinical guidance, the rationale behind this being that:

'Public health and clinical audiences share the same need for evidence-based, cost-effective solutions to the challenges in their day-to-day practice, as well as to inform policies and strategies to improve health'.

The guidelines for healthcare professionals have a strong focus on primary care in terms of both prevention and clinical care. The guidance is based around supporting and promoting behaviour change, with a strategy of getting all staff involved.

The key clinical guidance offered by NICE guideline 43 is summarised in Box 2. Multicomponent interventions are the treatment of choice including behavioural change strategies. The guidelines also suggest that when choosing treatments, the following factors should be considered:
- the person's individual preference and social circumstance and the experience and outcome of previous treatments (including whether there were any barriers);

- their level of obesity and risk, based on BMI and waist circumference (Box 2); and

- comorbidities.

Thus, a person who is classified as overweight but not obese, with a low waist circumference can be offered general advice on healthy weight and lifestyle; a person who is overweight or has grade 1 obesity with high or very high waist circumference can be offered diet and physical activity advice; a person who has grade 2 obesity (or overweight/grade 1 obesity with comorbidities) offered diet and physical activity advice plus considered for weight loss drugs; and a person with grade 3 obesity (or grade 2 obesity with comorbidities) offered diet and physical activity advice plus considered for weight loss drugs and surgery.

Details of the different approaches to diet and exercise, and the behavioural methods that may help are fully described in the guidelines. In general for an adult, diets that have a $600 \mathrm{kcal} /$ day deficit (that is, they contain $600 \mathrm{kcal}$ less than the person needs to stay the same weight) or that reduce calories by lowering the fat content (low-fat diets), in combination with expert support and intensive follow-up, are recommended for sustainable weight loss. In terms of exercise, NICE advises that adults should be encouraged to do at least 30 minutes of at least moderate-intensity physical

\section{Box 1. NICE Clinical Guideline 43.}

\section{Obesity: the prevention, identification, assessment, and management of overweight and obesity in adults and children}

The guidance covers:

- how staff in GP surgeries and hospitals should assess whether people are overweight or obese;

- what staff in GP surgeries and hospitals should do to help people lose weight;

- care for people whose weight puts their health at risk;

- how people can make sure they and their children stay at a healthy weight; and

- how health professionals, local authorities and communities, childcare providers, schools, and employers should make it easier for people to improve their diet and become more active.

The guidance aims to:

- stem the rising prevalence of obesity and diseases associated with it;

- increase the effectiveness of interventions to prevent overweight and obesity; and

- improve the care provided to adults and children with obesity, particularly in primary care.

Key priorities for implementation are:

1. prevention;

2. management in children; and

3. management in adults. 


\section{Box 2. Summary of key clinical guidance offered by NICE guideline 43.}

For adults, NICE guideline 43 includes guidance on:

- Assessment:

- BMI/waist circumference in addition if $\mathrm{BMI}<35 \mathrm{~kg} / \mathrm{m}^{2}$.

- Lifestyle, comorbidities, willingness to change.

- Advice on information giving.

- Offering multi-component interventions to increase physical activity, increase healthy eating, and improve eating behaviour.

- Drugs:

Discussing with the patient the potential benefits and limitations, including the mode of action, adverse effects, and monitoring requirements and their potential impact on the patient's motivation. When drug treatment is prescribed, arrangements should be made for appropriate health professionals to offer information, support, and counselling on additional diet, physical activity, and behavioural strategies. Information about patient support programmes should also be provided.

- General principles of long-term follow-up by trained professional. Continuity of records and interventions tailored to individuals needs.

- Referral criteria.

- Surgery

- Bariatric surgery is recommended as a treatment option for adults with obesity if all of the following criteria are fulfilled:

- they have a BMI of $>40 \mathrm{~kg} / \mathrm{m}^{2}$, or $35-40 \mathrm{~kg} / \mathrm{m}^{2}$ and other significant disease (for example, type 2 diabetes or high blood pressure) that could be improved if they lost weight;

- all appropriate non-surgical measures have been tried but have failed to achieve or maintain adequate, clinically beneficial weight loss for at least 6 months;

- the person has been receiving or will receive intensive management in a specialist obesity service;

- the person is generally fit for anaesthesia and surgery; and

- the person commits to the need for long-term follow-up.

Bariatric surgery is also recommended as a first-line option for adults with a BMI of $>50 \mathrm{~kg} / \mathrm{m}^{2}$ in whom surgical intervention is considered appropriate.

For children the guidance also includes advice on:

- Targetting family and social setting.

- Information giving.

- BMl age and sex adjusted.

- Tailored interventions if BMI above 90th centile, consider assessing for comorbidities if above 97th centile.

- Assessment of lifestyle, comorbidities, and willingness to change.

- Offering multi-component interventions.

- General principles of long-term follow-up, continuity of records, and encouragment of parental responsibility.

- Considering specialist management (drugs/surgery) for those with significant comorbidity or complex needs.

activity on $\geq 5$ days a week. The activity can be in one session or several lasting 10 minutes or more. To prevent obesity, most people should be advised they may need to do 45-60 minutes of moderateintensity activity a day, particularly if they do not reduce their energy intake. People who have been obese and have lost weight should be advised they may need to do $60-90$ minutes of activity a day to avoid regaining weight.

\section{WHAT HAS HAPPENED SINCE NICE CLINICAL GUIDELINE 43?}

There have been some updates to the guideline since publication in 2006 (expected review date: November 2011). Other guidelines published since 2006 include the European Clinical Practice Guidelines for the management of obesity in adults published in $2008,{ }^{4}$ the Interdisciplinary European guidelines on surgery of severe obesity, ${ }^{5}$ and the Canadian clinical practice guidelines on the management and prevention of obesity in adults and children. ${ }^{6}$ The Scottish Intercollegiate Guidelines Network (SIGN), which first published guidelines on obesity management in 1996, are in the process of updating their guidelines and are due to publish combined adult and child guidelines in 2010 .

There have also been a number of Cochrane systematic reviews on obesity published since 2006, including interventions for obesity in children, ${ }^{7}$ surgery for obesity in adults, ${ }^{8}$ intragastric balloon treatment in adults, ${ }^{9}$ effectiveness of chitosan (a dietary supplement reported to reduce body weight), ${ }^{10}$ the long-term effectiveness of pharmacotherapy for obesity and overweight, ${ }^{11}$ and the long-term effects of weight reducing drugs in hypertensive patients. ${ }^{12}$ 
Collectively these newer guidelines and reviews add little to the key messages delivered in the 2006 NICE guideline 43. Gastric surgery, however, would appear to be gaining an increasing research focus, with growing evidence that it is generally more effective than conventional treatments in moderate and severe obesity although this needs to be balanced against postoperative complications and deaths. In terms of long-term effects of weight reducing drugs, orlistat, sibutramine and rimonabant have been studied in trials of 1 year or longer, but internal validity of studies was limited by high attrition rates. All three anti-obesity agents are modestly effective in reducing weight and have differing effects on cardiovascular risk and adverse effects profiles. Longer and more methodologically rigorous studies of anti-obesity drugs that are powered to examine endpoints such as mortality and cardiovascular morbidity are required.

\section{HOW USEFUL ARE CLINICAL GUIDELINES FOR THE MANAGEMENT OF OBESITY IN GENERAL PRACTICE AND PRIMARY CARE?}

Weight management guidelines and the first law of thermodynamics

The first law of thermodynamics states that energy can be transformed (changed from one form to another), but cannot be created or destroyed..$^{13}$ In the context of humans or other animals this means that the energy stored by the body must equal (over time) energy intake minus energy expenditure. This simple law of nature, often referred to as the energy balance equation, means that interventions aiming to reduce weight must either reduce calorie intake (metabolisable energy intake), increase energy expenditure (in practical terms by exercise), or both. NICE and all other past and future guidelines on weight management are thus bound by this equation. It is not surprising then that NICE guideline 43 and other guidelines on weight management and obesity contains evidence reviews across these key components of diet and exercise, and behavioural change methods that may help either or both.

\section{How relevant are the NICE guidelines on obesity to UK primary care?}

The NICE guidelines are the most comprehensive and thorough of recent guidelines, with the full guideline running to a daunting 1942 pages (which presumably few GPs or primary care staff have the time or energy to read). However, the summary guidelines for healthcare professionals offer a much more manageable information source, and NICE have gone to extraordinary lengths to make these guidelines accessible to a very wide audience, including the public. NICE have also issued guidance on how to try to implement guidelines into practice. Thus, on face value, NICE guideline 43 is extremely relevant and potentially useful in the fight against obesity from a public health and primary care perspective.

However, reading the full guidelines plus the evidence summaries that underpin them reveals some interesting facts. In terms of reducing energy intake (dietary manipulation) the recommendations are based on findings from 29 studies, but only two of these were conducted in the UK with the majority being carried out in the US. Similarly, for behaviour change, only two studies were conducted in the UK, and the majority were carried out in the US. The evidence on exercise was derived from 33 studies, 26 of which were based in the US, and none in the UK. Although one could argue that the first law of thermodynamics and the energy balance equation applies to all people irrespective of nationality, there are of course cultural differences between countries that may influence the outcomes of different interventions in different settings.

Perhaps more importantly, very few studies cited in NICE guideline 43 were actually conducted in primary care (two on diet, one on behaviour change, and two on exercise) and most studies were conducted on volunteers (presumably with high motivation to lose weight). As NICE guideline 43 concludes:

'It is difficult, therefore, to know how generalisable the results of the included studies are to the UK population, particularly in primary care. One assumption could be that the effect size achieved in the included studies may be smaller in practice.'

NICE also adds:

'The effectiveness of all interventions appears to change over time, with a trend for greater weight loss in the short term (up to 12 months), with a reduction in overall weight loss in the longer term (up to 60 months).' [Evidence Grade 1++].

Given that the NICE guidelines are developed for the UK healthcare system, and a key stated aim of NICE guideline 43 is to improve weight management in primary care (Box 1), one could question the relevance of many of the studies used to develop the guidelines to UK general practice and primary care. 


\section{Possible ways forward and future research}

Numerous studies have shown the problems of implementing guidelines in general practice and primary care, and there is no need to re-state this except to highlight a few key points. The most commonly reported barriers to effective treatment in the primary care setting ${ }^{14}$ include:

- psychological complexities of cases;

- high rate of relapse;

- perceived lack of effective interventions;

- lack of time;

- lack of resources; and

- lack of onward referral options.

A qualitative study carried out on GPs and practice nurses in Scotland found similar barriers and concluded that:

'A comprehensive and integrative primary care-led approach to weight management may be possible but will need substantial shifts of resources, organisation, training, and attitudes in order to maximise its potential impact. ${ }^{, 15}$

In this issue of the Journal, Turner and colleagues report similar views from primary care practitioners in terms of childhood obesity. ${ }^{16}$ Also in this issue, Winzenberg and colleagues report that, rather than assessing physical exercise in all patients, GPs vary their approach depending on the clinical and social context. ${ }^{17}$ This is perhaps unsurprising given that many patients with obesity have other comorbid long-term conditions (related or unrelated to obesity). In a recent UK primary care study, $74 \%$ of obese patients had one or more comorbid conditions, almost half had two or more, and weight loss was less in those with comorbidities such as diabetes and arthritis. ${ }^{18}$ One way forward could be the recognition that the management of a complex condition such as obesity requires complex interventions. ${ }^{19}$ Given that evidence-based guidelines are based on studies that largely exclude patients with multiple morbidity, the management of obesity may benefit from studies specifically targeted at patients with multimorbidity. ${ }^{20-23}$

Obesity is socially patterned, with higher levels of obesity (with more multimorbidity) in areas of high socioeconomic deprivation, but with services that do not match need. ${ }^{24}$ There is a dearth of research on obesity management in deprived areas, or on specific interventions for people of differing socioeconomic status. In one study quoted in NICE guideline 43, a subgroup analysis found that a low-carbohydrate/low-fibre diet tended to be more successful for weight loss among people in a lower social class (classes III-IV) than a higher-carbohydrate/higher-fibre diet. ${ }^{25}$ Clearly, more targeted research may be helpful, especially given recent evidence suggesting effectiveness of low carbohydrate diets. ${ }^{25-27}$

Social interventions aimed at tackling obesity also offer an alternative interesting approach. ${ }^{28}$ Evidence from interventions in 'real life' primary care are also much needed and approaches such as 'Counterweight' in the UK are being watched with interest. ${ }^{18}$

In conclusion, obesity management is influenced by a huge range of human, system, and environmental factors. As NICE guideline 43 states:

'It is unlikely that the problem of obesity can be addressed through primary care management alone ... the clinical management of obesity cannot be viewed in isolation from the environment in which people live.'

Tackling the social determinants of health and the obesogenic environment must be top priorities. However, primary care clearly has an important role to play, but in such a complex condition as obesity, clinical guidelines alone cannot lead patient and practitioner through the labyrinth of decisions that need to be made in prioritising and targeting which problem to address first and how. Individualised care, clinical wisdom, and decisions based on a trusting relationship can, however, perhaps begin to tackle such complexity, ${ }^{29}$ and the traditional holistic strengths of general practice in the UK population coverage and contact, relational continuity and empathic relationships, and an ability to deal with complexity ${ }^{30}$ - may offer part of the solution if the additional resources and support required are made available.

\section{Competing interests}

The author has stated that there are none.

\section{Discuss this article}

Contribute and read comments about this article on the Discussion Forum: http://www.rcgp.org.uk/bjgp-discuss

\section{REFERENCES}

1. NICE. Obesity: guidance on the prevention, identification, assessment and management of overweight and obesity in adults and children. London: National Institute for Health and Clinical Excellence, 2006. http://www.nice.org.uk/CG43 (accessed 5 Oct 2009).

2. Rothman AA, Wagner EH. Chronic illness management: what is the role of primary care? Ann Intern Med 2003; 138(3): 256-261.

3. The NHS Confederation (Employers) Company Ltd. Quality and Outcomes Framework Guidance for GMS Contract 2009/2010. Delivering investment in general practice.

http://www.nhsemployers.org/Aboutus/Publications/

Documents/QOF_Guidance_2009_final.pdf (accessed 5 Oct 2009).

4. Tsigos C, Hainer V, Basdevant A, et al. Management of obesity in 
adults: European Clinical Practice Guidelines. Obesity Management Task Force of the European Association for the Study of Obesity. Obesity Facts 2008; 1: 106-116.

5. Fried M, Hainer V, Basdevant A, et al. Interdisciplinary European guidelines on surgery of severe obesity. Obesity Facts 2008; 1: $52-58$.

6. Lau DCW, Douketis JD, Morrison KM, et al. 2006 Canadian clinical practice guidelines on the management and prevention of obesity in adults and children. Executive summary. CAMJ 2007; 176(8): S1-13.

7. Oude Luttikhuis $\mathrm{H}$, Baur L, Jansen $\mathrm{H}$, et al. Interventions for treating obesity in children. Cochrane Database Syst Rev 2009; 1: CD001872. DOI: 10.1002/14651858.CD001872.pub2.

8. Colquitt JL, Picot J, Loveman E, Clegg AJ. Surgery for obesity. Cochrane Database Syst Rev 2009; 2: CD003641. DOI: 10.1002/14651858.CD003641.pub3.

9. Fernandes MAP, Atallah ÁN, Soares B, et al. Intragastric balloon for obesity. Cochrane Database Syst Rev 2007; 1: CD004931. DOI: 10.1002/14651858.CD004931.pub2.

10. Jull AB, Ni Mhurchu C, Bennett DA, et al. Chitosan for overweigh or obesity. Cochrane Database Syst Rev 2008; 3: CD003892. DOI: 10.1002/14651858.CD003892.pub3.

11. Padwal RS, Rucker D, Li SK, et al. Long-term pharmacotherapy for obesity and overweight. Cochrane Database Syst Rev 2009; 4: CD004094. DOI: 10.1002/14651858.CD004094.pub2.

12. Siebenhofer A, Horvath K, Jeitler K, et al. Long-term effects of weight-reducing drugs in hypertensive patients. Cochrane Database Syst Rev 2009; 3: CD007654. DOI: 10.1002/14651858.CD007654.pub2

13. Wikipedia. First Law of Thermodynamics. http://en.wikipedia.org/wiki/First law of thermodynamics (accessed 7 Oct 2009).

14. Maryon-Davis A. Weight management in primary care: how can it be made more effective? Proc Nutr Soc 2005; 64(1): 97-103.

15. Mercer SW, Tessier S. A qualitative study of GP's and practice nurse's attitudes to obesity management in primary care. Health Bulletin 2001; 59(4): 248-253.

16. Turner KM, Shiled JPH, Salisbury C. Practitioners' views on managing childhood obesity in primary care: a qualitative study. $\mathrm{Br}$ J Gen Pract 2009; 59: 856-862.

17. Winzenberg T, Reid P, Shaw K. Assessing physical activity in general practice: a disconnect between clinical practice and public health?
Br J Gen Pract 2009; 59: 850-855.

18. The Counterweight Project Team. Evaluation of the Counterweigh Programme for obesity management in primary care: a starting point for continuous improvement. Br J Gen Pract 2008; 58(553) 548-554.

19. Campbell NC, Murray E, Darbyshire J, et al. Designing and evaluating complex interventions to improve health care. $B M J$ 2007; 334(7591): 455-459.

20. Fortin M, Hudon C, Lapointe L, Vanasse A. Multimorbidity is common to family practice: is it commonly researched? Can Fam Physician 2005; 51: 244-245.

21. Fortin M, Soubhi H, Hudon C, et al. Multimorbidity's many challenges. BMJ 2007; 334(7602): 1016-1017.

22. Smith SM, O'Dowd T. Chronic diseases: what happens when they come in multiples? Br J Gen Pract 2007; 57(537): 268-270.

23. Mercer SW, Smith SM, Wyke S, et al. Multimorbidity in primary care: developing the research agenda. Fam Pract 2009; 26(2): 79-80.

24. Mercer SW, Watt GMC. The inverse care law: clinical primary care encounters in deprived and affluent areas of Scotland. Ann Fam Med 2007; 5(6): 503-510.

25. Baron JA, Schori A, Crow B, et al. A randomized controlled trial of low carbohydrate and low fat/high fiber diets for weight loss. Am J Public Health 1986; 76(11): 1293-1296.

26. Nordmann AJ, Nordmann A, Briel M, et al. Effects of lowcarbohydrate vs low-fat diets on weight loss and cardiovascular risk factors: a meta-analysis of randomized controlled trials. Arch Intern Med 2006; 166(3): 285-293.

27. Gardner CD, Kiazand A, Alhassan S, et al. Comparison of the Atkins, Zone, Ornish, and LEARN diets for change in weight and related risk factors among overweight premenopausal women: the A TO Z Weight Loss Study: a randomized trial. JAMA 2007; 297(9): 969-977.

28. Woodman J, Lorenc T, Harden A, Oakley A. Social and environmental interventions to reduce childhood obesity: a systematic map of reviews. London: EPPI-Centre, Social Science Research Unit, Institute of Education, University of London, 2008.

29. Mercer SW, Lean MEJ. Request for slimming tablets. BMJ 2008; 337: a1287. doi: 10.1136/bmj.a1287.

30. Gillies J, Mercer SW, Lyon A, et al. Distilling the essence of general practice: a learning journey in progress. Br J Gen Pract 2009, 59(562): $167-176$ 